Revista Verde de Agroecologia e Desenvolvimento Sustentável

http://www.gvaa.com.br/revista/index.php/RVADS

ARTIGO CIENTÍFICO

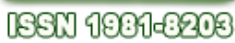

DOI: http://dx.doi.org/10.18378/rvads.v9i4.2951

\title{
Estudo comparativo entre acessos e cultivares de manjericão no Estado do Tocantins
}

\section{Comparative study between basil accessions and cultivars in the Tocantins State}

\author{
Ronice Alves Veloso ${ }^{1 *}$, Henrique Guilhon de Castro ${ }^{2}$, Dione Pereira Cardoso ${ }^{3}$, Aloisio Freitas Chagas Júnior ${ }^{2}$, Lillian França \\ Borges Chagas ${ }^{1}$.
}

Resumo: O objetivo deste trabalho foi avaliar o crescimento e a produção de biomassa de quatro acessos, provenientes de diferentes regiões geográficas, e duas cultivares comerciais de manjericão (Ocimum basilicum L.) cultivados sob as mesmas condições edafoclimáticas no Estado do Tocantins. Os acessos foram coletados no município de Gurupi-TO (GUR) e de Monte Alegre-GO (MAL, MVE e FPS). As sementes da cultivar comercial manjericão roxo (MR) foram adquiridas no comércio local de Gurupi-TO e as sementes da cultivar Maria Bonita (MB) foram fornecidas pelo Horto de Plantas Medicinais da Universidade Federal de Lavras. As avaliações foram realizadas em intervalos regulares de 15 dias, com avaliação da altura das plantas, diâmetro do caule, número de inflorescências e vigor das plantas. Na última época de avaliação, foram avaliadas as características de massa fresca total, massa fresca das folhas, massa fresca das inflorescências, massa fresca dos ramos, número de folhas, número de inflorescências, número de ramos, massa seca total das folhas, massa seca total das inflorescências, massa seca total dos ramos e massa seca total. Nas cultivares MB e MR foi obtida maior taxa de crescimento em altura e maior vigor em relação aos outros acessos. Nas cultivares MB e MR observaram os maiores valores de massa fresca total e massa seca total. Nos acessos silvestres (MAL, GUR, FPS e MVE) foi observado maior número de inflorescências e maior número de folhas.

Palavras-chaves: Plantas medicinais, Ocimum basilicum, análise do crescimento, produtividade.

\begin{abstract}
The objective of this study was to evaluate the growth and biomass production from four wild accessions of basil (Ocimum basilicum L.) from different geographical regions, and two commercial cultivars grown under the same edaphoclimatic conditions in the conditions of Tocantins State. The accessions were collected in the county of Gurupi-TO (GUR) and Monte Alegre-GO (MAL, MVE and FPS). The seeds of the commercial cultivar purple basil (MR) were acquired in market of Gurupi-TO and the seeds of the cultivar Maria Bonita (MB) were provided by Botanic Garden of Medicinal Plants of Federal University of Lavras. The evaluations were performed at regular intervals of 15 days, with evaluation of plants height, diameter of stem, number of inflorescences and vigor of plants. In the last evaluation time, the characteristics of total fresh mass, fresh mass leaves, fresh mass of inflorescences, fresh mass of branches, number of leaves, number of inflorescences, number of branches, total dry mass of leaves, total dry mass of inflorescences, total dry mass of branches and total dry mass were measured. In the cultivars MB and MR were obtained the highest growth rate in height and greater vigor in relation to other accessions. In the cultivars MB and MR observed the highest values of total fresh mass and total dry mass. In the wild accessions (MAL, GUR, FPS and LVM) was observed higher number of inflorescences and higher number of leaves.
\end{abstract}

Key words: Medicinal plants, Ocimum basilicum, growth analysis, productivity.

\footnotetext{
*Autor para correspondência

Recebido para publicação em 25/09/2014; aprovado em 22/12/2014.

${ }^{1}$ Doutoranda em Produção Vegetal na Universidade Federal do Tocantins. Rua Badejós, Lote 7, Chácaras 69/72, Zona Rural, Caixa Postal 66, 77402-970 Gurupi - TO, E-mails: ronicealves@ hotmail.com e lillianfb@hotmail.com

${ }^{2}$ Professores Associados na Universidade Federal do Tocantins. Rua Badejós, Lote 7, Chácaras 69/72, Zona Rural, Caixa Postal 66, 77402-970 Gurupi - TO,

E-mails: hguilhon@uft.edu.br e chagasjraf@uft.edu.br

${ }^{3}$ Bolsista Prodoc/Capes na Universidade Federal do Tocantins. Rua Badejós, Lote 7, Chácaras 69/72, Zona Rural, Caixa Postal 66, 77402-970 Gurupi - TO, Email: cardoso.dione@gmail.com.
} 


\section{INTRODUÇÃO}

A disponibilidade de informações abordando aspectos fitotécnicos para produção agrícola de plantas medicinais, aromáticas e condimentares são limitadas. Assim, a obtenção de matéria-prima em espécies medicinais necessita de estudos que revelam o comportamento dessas espécies quando submetidas a técnicas de cultivo. A análise de crescimento pode indicar as diferenças entre variedades da mesma espécie, de forma a selecionar aquelas que melhor atendam aos objetivos propostos da exploração econômica (CASTRO et al., 1999; CASTRO et al., 2006).

O desenvolvimento de técnicas agronômicas que maximizam o cultivo de espécies medicinais é uma forma de assegurar a quantidade e a regularidade do fornecimento de matéria-prima, controlando os fatores que influenciam na sua qualidade fitoquímica e farmacológica, garantindo o fornecimento de princípios ativos de excelência (CHAGAS et al., 2011; LOURENZANI et al., 2004; PINTO et al., 2008).

A produção de biomassa é um dos fatores que restringem a produção de princípios ativos de interesse econômicos, uma vez que são nas folhas que ocorrem a maior produção de óleos essenciais (MAY et al., 2008). A falta de domínio tecnológico de todas as etapas de desenvolvimento das plantas medicinais pode levar à baixa qualidade da biomassa e de teores dos principais constituintes químicos do óleo essencial e de rendimentos (CHAGAS et al., 2011). Segundo Blank et al. (2005), antes de se iniciar o cultivo em escala comercial, é necessário conhecer o comportamento da espécie com relação aos efeitos climáticos da região de plantio, aos tratos culturais e aos fatores bióticos responsáveis pelo desenvolvimento da planta.

O manjericão (Ocimum basilicum L.) é uma planta herbácea, pertencente à família Lamiaceae, fortemente aromática, que pode ser anual ou perene, dependendo do local em que é cultivado. É originário do sudoeste Asiático e da África central, e se adaptou bem as condições climáticas brasileiras, podendo ser cultivado o ano todo. Apresenta grande valor econômico, pois é muito utilizada para diversos fins, como ornamental, condimentar, medicinal, aromática, na indústria farmacêutica e de cosméticos e para produção de óleo essencial (ROSADO et al., 2011; SILVA et al., 2005).

O objetivo deste trabalho foi avaliar o crescimento e a produção de biomassa de quatro acessos e duas cultivares de manjericão, provenientes de diferentes regiões geográficas, mas cultivados sob as mesmas condições edafoclimáticas, visando identificar os acessos ou as cultivares com maior adaptação e maior produção de biomassa nas condições do sul do Estado do Tocantins.

\section{MATERIAL E MÉTODOS}

Foram coletados quatro acessos de manjericão ( $O$. basilicum) em diferentes localidades e duas cultivares comerciais. Os acessos silvestres foram coletados nos

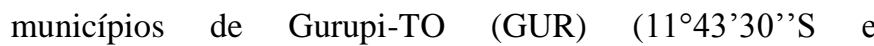
4904'34',W, $287 \mathrm{~m}$ ) e Monte Alegre-GO. No município de Monte Alegre foram coletados três acessos: o acesso MAL coletado no bairro Central (1315'24' S e 46 53 '24', 543 $\mathrm{m})$, o acesso MVE coletado no bairro Montes Verdes $\left(13^{\circ} 15^{\prime} 36^{\prime}\right.$ ' S e $\left.46^{\circ} 52^{\prime} 53^{\prime}, \mathrm{W}, 548 \mathrm{~m}\right)$ e o acesso FPS coletado na fazenda Ponta da Serra (1313'05' S e $46^{\circ} 48^{\prime} 24^{\prime \prime}$ W, $\left.577 \mathrm{~m}\right)$. Para a cultivar comercial denominada manjericão roxo (MR) as sementes foram adquiridas no comércio local do município de Gurupi-TO e as sementes da cultivar Maria Bonita (MB) foram fornecidas pelo Horto de Plantas Medicinais da Universidade Federal de Lavras.

As exsicatas dos acessos coletados foram depositadas no herbário da Universidade Federal de Lavras com os números: MAL - 26.599; GUR - 26.603; FPS - 26.602; MVE - 26.601; MB - 26.920; e MR - 26.600.

O experimento foi conduzido em casa de vegetação na Universidade Federal do Tocantins, câmpus de Gurupi, localizado a $11^{\circ} 43^{\prime} \mathrm{S}$ e $49^{\circ} 04^{\prime} \mathrm{W}$, com altitude média de 300 $\mathrm{m}$. Utilizou-se o delineamento experimental inteiramente casualizado, em esquema de parcela subdividida, com quatro repetições. As parcelas foram constituídas por quatro acessos e duas cultivares de manjericão e as subparcelas por cinco épocas de amostragem com intervalos regulares de quinze dias. A unidade experimental foi constituída por dois vasos e em cada vaso duas plantas, totalizando 24 plantas por repetição.

A análise química do substrato utilizado no experimento apresentou a seguinte composição química: $\mathrm{pH}\left(\mathrm{H}_{2} \mathrm{O}\right)=7,6$; $\mathrm{H}^{+}+\mathrm{Al}^{3+}=0,99 \mathrm{cmol}_{\mathrm{c}} \mathrm{dm}^{-3} ; \mathrm{Ca}^{2+}=2,73 \mathrm{cmol}_{\mathrm{c}} \mathrm{dm}^{-3} ; \mathrm{Mg}^{2+}=$ $2,14 \mathrm{cmol}_{\mathrm{c}} \mathrm{dm}^{-3} ; \mathrm{P}($ extrator Melich 1$)=107,4 \mathrm{mg} \mathrm{dm}^{-3} ; \mathrm{K}^{+}=$ $798,4 \mathrm{mg} \mathrm{dm}^{-3}$; matéria orgânica $=66,64 \mathrm{~g} \mathrm{dm}^{-3} ; \mathrm{CTC}=6,92$ $\mathrm{cmol}_{\mathrm{c}} \mathrm{dm}^{-3}$ e V\%=87,48\%.

O plantio das sementes foi realizado em vasos com capacidade de dez litros, utilizando-se como substrato solo de barranco mais esterco bovino na proporção 2:1. Aos 88 dias após o plantio foi efetuada a adubação de cobertura, utilizando-se $170 \mathrm{~g}$ de esterco bovino curtido em cada vaso.

$\mathrm{Na}$ análise do crescimento das plantas as avaliações foram realizadas em intervalos regulares de quinze dias, sendo a primeira avaliação realizada aos 42 dias após o plantio. Foram avaliadas as características de altura das plantas, diâmetro do caule, número de inflorescências e vigor das plantas. Para determinação do vigor de plantas foi adotado uma escala de notas de um a quatro considerando o desenvolvimento da parte aérea e arquitetura da planta.

Na última época de avaliação, aos 102 dias após o plantio, as plantas foram coletadas realizando-se o corte rente ao solo e determinadas as seguintes características: massa fresca total, massa fresca das folhas, massa fresca das inflorescências, massa fresca dos ramos, número de folhas, número de inflorescências, número de ramos, massa seca das folhas, massa seca das inflorescências, massa seca dos ramos e massa seca total.

Também foi realizada a determinação do comprimento e largura média das folhas de cada acesso. Desta forma utilizou-se de cada acesso uma planta como amostra, da qual foram retiradas 40 folhas de diferentes tamanhos (folhas de tamanho grande, médio, intermediário e pequeno) para mensuração do comprimento e largura média das folhas.

Para avaliar a massa seca, amostras de $20 \mathrm{~g}$ de cada planta foram mantidas em estufa com circulação forçada de ar em $70^{\circ} \mathrm{C}$ por 72 horas até massa constante.

Os dados foram interpretados por meio de análises de variância e de regressão. As médias foram comparadas pelo teste de Tukey a $5 \%$ de probabilidade e as equações de regressão foram ajustadas com base no teste " $\mathrm{t}$ " dos coeficientes a 5 ou $1 \%$ de probabilidade e no coeficiente de 
determinação. As análises estatísticas foram feitas no programa SAEG (Sistema para Análises Estatísticas e Genéticas) (RIBEIRO JÚNIOR \& MELO, 2009).

\section{RESULTADOS E DISCUSSÃO}

Nas Tabelas 1, 3 e 4 é mostrado o resumo das análises de variância na análise de crescimento e na análise destrutiva.

Tabela 1. Resumo das análises de variância das variáveis: altura (ALT), diâmetro do caule (DC), vigor (VIG) e número de inflorescências (NI) de quatro acessos e de duas cultivares de manjericão, em função de cinco épocas de amostragem.GurupiTO, 2012.

\begin{tabular}{|c|c|c|c|c|c|}
\hline \multirow[b]{2}{*}{ FV } & \multirow[b]{2}{*}{ GL } & \multicolumn{4}{|c|}{ Quadrado Médio } \\
\hline & & ALT & DC & VIG & NI \\
\hline Acessos (AC) & 5 & $615,5525 * *$ & $80,48020 * *$ & $1,980613 * *$ & $55599,00 * *$ \\
\hline Épocas (EP) & 4 & $10158,13 * *$ & $233,4037 * *$ & $1,906250 * *$ & $225267,6 * *$ \\
\hline $\mathrm{AC} \times \mathrm{EP}$ & 20 & $193,1349 * *$ & $4,986018 * *$ & $0,264931^{*}$ & $7658,186^{* *}$ \\
\hline Resíduo & 90 & 12,31578 & 1,052700 & 0,138175 & 1744,616 \\
\hline $\mathrm{CV} \%$ & & 6,764 & 15,134 & 10,324 & 43,108 \\
\hline
\end{tabular}

Tabela 3. Resumo das análises de variância das variáveis massa fresca total (MFT), massa fresca das folhas (MFF), massa fresca das inflorescências (MFI), massa fresca dos ramos (MFR), número de folhas (NF) e número de inflorescências (NI), de quatro acessos e de duas cultivares de manjericão (AC).Gurupi-TO, 2012.

\begin{tabular}{lccccccc}
\hline \multicolumn{7}{c}{ Quadrado Médio } \\
\hline FV & GL & MFT & MFF & MFI & MFR & NF & NI \\
\hline AC & 5 & $238717,9^{* *}$ & $69636,07^{* *}$ & $1954,77^{* *}$ & $33868,79^{* * *}$ & $3467480^{\text {ns }}$ & $34636,90^{* *}$ \\
Res. & 18 & 11445,30 & 2243,16 & 365,95 & 2363,29 & 2574114 & 7038,40 \\
\hline CV\% & & 24,48 & 24,40 & 24,63 & 29,42 & 36,34 & 33,83 \\
\hline
\end{tabular}

** = significativo a $1 \%$ de probabilidade pelo teste $\mathrm{F} ; \mathrm{ns}=$ não significativo a $5 \%$ de probabilidade pelo teste $\mathrm{F}$.

Tabela 4. Resumo das análises de variância das variáveis número de ramos (NR), massa seca total das folhas (MSTF), massa seca total das inflorescências (MSTI), massa seca total dos ramos (MSTR), massa seca total (MST), de quatro acessos e de duas cultivares de manjericão (AC).Gurupi-TO, 2012.

\begin{tabular}{lcccccc}
\hline FV & GL & NR & MSTF & MSTI & MSTR & MST \\
\hline AC & 5 & $47849,01^{\text {ns }}$ & $1460,24^{* *}$ & $706,64^{* *}$ & $1460,71^{* *}$ & $5390,26^{* *}$ \\
Res. & 18 & 50339,08 & 92,38 & 82,46 & 231 & 857,12 \\
\hline CV\% & & 43,72 & 26,80 & 25,92 & 33,69 & 23,62 \\
\hline
\end{tabular}

Nas condições que foi realizado o trabalho observou-se que as cultivares MB e MR apresentaram melhor performance de vigor e de produção de biomassa. Entretanto, devem ser realizados outros experimentos de campo para obter dados consistentes na adaptação destas cultivares as condições edafoclimáticas do Estado do Tocantins. Os acessos silvestres devem ser preservados, pois são fontes de genes e podem ser utilizados em futuros programas de melhoramento genético do manjericão.

Em todos os acessos e cultivares avaliados observou-se que a altura aumentou linearmente em função do tempo. A cultivar MR apresentou maiores taxas de crescimento em altura de plantas a cada intervalo de tempo, $1,2606 \mathrm{~cm} \mathrm{dia}^{-1}$, atingindo aos 102 dias após o plantio $96,8302 \mathrm{~cm} \mathrm{planta}^{-1}$. A menor taxa de crescimento em altura a cada intervalo de tempo foi observado no acesso GUR com 0,6314 $\mathrm{cm} \mathrm{dia}^{-1}$ (Tabela 2). Luz et al. (2009) avaliaram dois genótipos de manjericão e constataram que não foi observada diferença significativa entre os genótipos quanto a variável altura; que a altura média das plantas foi de 49,9 cm em um dos genótipos e de 41,6 cm no outro genótipo.
Na variável diâmetro do caule (Tabela 2), a cultivar MB se destacou com aumento de $0,2155 \mathrm{~mm} \mathrm{dia}^{-1}$, passando de $3,9514 \mathrm{~mm}$ aos 42 dias após o plantio para $16,8814 \mathrm{~mm}$ na última avaliação. Na primeira avaliação não houve diferença entre os acessos analisados, no entanto nas épocas de avaliações posteriores, a cultivar MB apresentou maior valor na variável diâmetro do caule. Blank et al. (2007) avaliaram a cultivar Maria Bonita e observaram que o diâmetro médio do caule foi de 13,2 mm. Blank et al. (2004) obtiveram para o diâmetro do caule de acessos de manjericão valores iguais ou superiores a $10 \mathrm{~mm}$.

Quanto ao vigor, não foi observada diferença $(\mathrm{P}>0,05)$ entre os acessos na terceira e quarta época de avaliação. Nos acessos GUR, FPS e MVE foram ajustados modelos quadráticos, apresentando padrões diferentes de crescimento em relação aos outros acessos. A cultivar MB mostrou boa adaptação às condições de cultivo, apresentando em todas as épocas de avaliação os maiores valores de vigor de plantas (Tabela 2). 
Tabela 2. Valores médios, equações de regressão e coeficiente de determinação de quatro acessos e de duas cultivares de manjericão nas variáveis: altura, diâmetro do caule, vigor e número de inflorescências, em cinco épocas de amostragem. Gurupi-TO, 2012.

\begin{tabular}{|c|c|c|c|c|c|c|c|}
\hline & \multicolumn{5}{|c|}{ Épocas de amostragem (dias após plantio) } & \multirow[b]{2}{*}{ Equações de regressão } & \multirow[b]{2}{*}{$\mathbf{r}^{2}$} \\
\hline & 42 & 57 & 72 & 87 & 102 & & \\
\hline \multicolumn{8}{|c|}{ Altura (cm planta $\left.{ }^{-1}\right)$} \\
\hline MAL & $26,550 \mathrm{a}$ & 43,075 a & $55,981 \mathrm{bc}$ & $63,750 \mathrm{~b}$ & $68,738 \mathrm{~b}$ & $\mathrm{y}=+1,1948+0,7003 \mathrm{EP}^{* *}$ & 0,9354 \\
\hline GUR & $23,375 \mathrm{ab}$ & $37,981 \mathrm{a}$ & $47,438 \mathrm{~d}$ & $56,825 \mathrm{~b}$ & $61,306 \mathrm{c}$ & $y=-0,0740+0,6314 \mathrm{EP}^{* *}$ & 0,9019 \\
\hline FPS & $23,313 \mathrm{ab}$ & 40,850 a & $53,731 \mathrm{~cd}$ & $60,531 \mathrm{~b}$ & $66,410 \mathrm{bc}$ & $y=-1,8539+0,7058 \mathrm{EP}^{* *}$ & 0,9391 \\
\hline MVE & $24,069 \mathrm{ab}$ & 40,481 a & $53,065 \mathrm{~cd}$ & $59,875 \mathrm{~b}$ & $63,594 \mathrm{bc}$ & $\mathrm{y}=+0,9637+0,6563 \mathrm{EP}^{* *}$ & 0,8928 \\
\hline MB & $21,231 \mathrm{ab}$ & 39,719 a & $61,856 \mathrm{ab}$ & 75,150 a & 92,619 a & $\mathrm{y}=-27,424+1,1880 \mathrm{EP}^{* * *}$ & 0,9675 \\
\hline MR & $19,313 \mathrm{~b}$ & $38,794 \mathrm{a}$ & $64,244 \mathrm{a}$ & $78,906 \mathrm{a}$ & $93,800 \mathrm{a}$ & $y=-31,751+1,2606 \mathrm{EP}^{* *}$ & 0,9756 \\
\hline \multicolumn{8}{|c|}{ Diâmetro do caule (mm planta $\left.{ }^{-1}\right)$} \\
\hline MAL & $2,654 \mathrm{a}$ & $4,038 \mathrm{~b}$ & $5,808 \mathrm{c}$ & $7,629 \mathrm{c}$ & $8,813 \mathrm{c}$ & $\mathrm{y}=-1,8481+0,1061 \mathrm{EP}^{* *}$ & 0,9490 \\
\hline GUR & $2,408 \mathrm{a}$ & $3,429 b$ & $5,311 \mathrm{c}$ & $6,956 \mathrm{c}$ & $8,253 \mathrm{c}$ & $\mathrm{y}=-2,0323+0,1014 \mathrm{EP}^{* *}$ & 0,9489 \\
\hline FPS & $2,468 \mathrm{a}$ & $3,960 \mathrm{~b}$ & $5,726 \mathrm{c}$ & $7,210 \mathrm{c}$ & $8,179 \mathrm{c}$ & $\mathrm{y}=-1,5349+0,0978 \mathrm{EP}^{* *}$ & 0,8920 \\
\hline MVE & $2,440 \mathrm{a}$ & $3,971 \mathrm{~b}$ & $6,189 \mathrm{bc}$ & $7,829 \mathrm{c}$ & $8,806 \mathrm{c}$ & $y=-2,1158+0,1106 \mathrm{EP}^{* *}$ & 0,9355 \\
\hline MB & $4,014 \mathrm{a}$ & $6,601 \mathrm{a}$ & $11,186 \mathrm{a}$ & $13,633 \mathrm{a}$ & $16,663 \mathrm{a}$ & $y=-5,0996+0,2155 \mathrm{EP}^{* *}$ & 0,8687 \\
\hline MR & $3,052 \mathrm{a}$ & $5,423 \mathrm{ab}$ & $8,189 \mathrm{~b}$ & $10,292 \mathrm{~b}$ & $12,258 \mathrm{~b}$ & $y=-3,3326+0,1552 \mathrm{EP}^{* *}$ & 0,9499 \\
\hline \multicolumn{8}{|c|}{ Vigor } \\
\hline MAL & $3,375 \mathrm{ab}$ & $3,688 \mathrm{ab}$ & 3,875 a & $3,750 \mathrm{a}$ & $3,333 \mathrm{ab}$ & $\mathrm{y}=+3,604$ & \\
\hline GUR & $2,563 \mathrm{c}$ & $2,750 \mathrm{c}$ & $3,750 \mathrm{a}$ & 3,813 a & $3,250 \mathrm{ab}$ & $\begin{array}{l}\mathrm{y}=-1,6082+0,1277 \mathrm{EP}^{* *}- \\
0,00077 \mathrm{EP}^{2 * *}\end{array}$ & 0,5022 \\
\hline FPS & $2,750 \mathrm{bc}$ & $3,188 \mathrm{bc}$ & 3,917 a & $3,197 \mathrm{a}$ & $3,438 \mathrm{ab}$ & $\begin{array}{l}\mathrm{y}=-1,4194+0,1312 \mathrm{EP}^{* *}- \\
0,00081 \mathrm{EP}^{2^{* *}}\end{array}$ & 0,4954 \\
\hline MVE & $2,875 \mathrm{bc}$ & $3,188 \mathrm{bc}$ & $3,875 \mathrm{a}$ & $3,750 \mathrm{a}$ & $3,229 \mathrm{~b}$ & $\begin{array}{l}\mathrm{y}=-0,9525+0,1218 \mathrm{EP}^{* *}- \\
0,00079 \mathrm{EP}^{2^{* *}}\end{array}$ & 0,5519 \\
\hline MB & $4,000 \mathrm{a}$ & $4,000 \mathrm{a}$ & $4,000 \mathrm{a}$ & $4,000 \mathrm{a}$ & $4,000 \mathrm{a}$ & $y=+4,000$ & \\
\hline MR & 3,938 a & $3,813 \mathrm{ab}$ & $4,000 \mathrm{a}$ & $4,000 \mathrm{a}$ & $4,000 \mathrm{a}$ & $y=+3,950$ & \\
\hline \multicolumn{8}{|c|}{ Número de inflorescências } \\
\hline MAL & $8,563 \mathrm{a}$ & 49,500 a & $130,188 \mathrm{a}$ & $195,063 \mathrm{a}$ & 344,688 a & $y=-246,95+5,45208 \mathrm{EP}^{* *}$ & 0,8412 \\
\hline GUR & $6,875 \mathrm{a}$ & $29,000 \mathrm{a}$ & $87,000 \mathrm{ab}$ & $146,125 \mathrm{a}$ & $241,938 \mathrm{bc}$ & $y=-179,69+3,91500 \mathrm{EP}^{* *}$ & 0,8282 \\
\hline FPS & $6,021 \mathrm{a}$ & $36,125 \mathrm{a}$ & $180,125 \mathrm{a}$ & $179,375 \mathrm{a}$ & $290,292 \mathrm{abc}$ & $y=-217,67+4,74528 \mathrm{EP}^{* *}$ & 0,7447 \\
\hline MVE & $7,438 \mathrm{a}$ & $43,125 \mathrm{a}$ & $138,000 \mathrm{a}$ & $220,417 \mathrm{a}$ & $313,021 \mathrm{ab}$ & $y=-234,06+5,25639 \mathrm{EP}^{* *}$ & 0,8803 \\
\hline MB & $0,000 \mathrm{a}$ & $00,000 \mathrm{a}$ & $1,063 \mathrm{~b}$ & $3,313 b$ & $213,313 \mathrm{c}$ & $\begin{array}{l}\mathrm{y}=+470,15- \\
16,388 \mathrm{EP}^{* *}+0,13371 \mathrm{EP}^{2 * *}\end{array}$ & 0,8332 \\
\hline MR & $0,000 \mathrm{a}$ & $00,000 \mathrm{a}$ & $5,563 \mathrm{~b}$ & $18,063 \mathrm{~b}$ & $84,625 \mathrm{~d}$ & $y=-68,260+1,22488 \mathrm{EP}^{* *}$ & 0,6256 \\
\hline
\end{tabular}

Médias seguidas pela mesma letra na coluna não diferem entre si pelo teste de Tukey (P > 0,05); ${ }^{* *}$ = significativo a $1 \%$ de probabilidade pelo teste " $t$ ". MAL Monte Alegre-GO; GUR - Gurupi-TO; FPS - Faz. Ponta da Serra; MVE - Montes Verdes; MB - Cv. Maria Bonita; e MR - Manjericão Roxo -Comercial.

De acordo com observações de campo nas cultivares Maria Bonita e Manjericão Roxo observou-se que o tamanho das folhas era maior que dos acessos silvestres com largura média de $2,45 \mathrm{~cm}$ e comprimento médio de $4,85 \mathrm{~cm}$. Nos acessos silvestres a largura média das folhas e o comprimento médio das folhas foram de $1,2 \mathrm{~cm}$ e $2,33 \mathrm{~cm}$, respectivamente. Nos acessos silvestres observou-se coloração branca das flores em todos os acessos (MAL, GUR, FPS e MVE), reforçando a similaridade entre os mesmos.

Observou-se que os acessos MAL, GUR, FPS e MVE (acessos com folhas estreitas) apresentaram florescimento precoce, aos 42 dias após o plantio, e as cultivares MB e MR (cultivares com folhas largas) florescimento tardio, com o surgimento dos primeiros racemos a partir da terceira avaliação, aos 72 dias após o plantio (Tabela 2). Resultado semelhante foi observado por Fernandes et al. (2004) ao avaliarem duas espécies de manjericão constataram que o início do florescimento do manjericão de folha larga foi tardio em relação ao início do florescimento do manjericão de folha estreita.

No número de inflorescências observou-se que apenas na cultivar MB foi ajustado o modelo quadrático, apresentando padrão de crescimento diferente dos demais acessos. Não houve diferença $(\mathrm{P}>0,05)$ entre os acessos na primeira e segunda avaliação. $\mathrm{O}$ acesso MAL apresentou a maior taxa de crescimento na variável número de inflorescências, 5,45208 racemos $\mathrm{dia}^{-1}$, atingindo aos 102 dias após o plantio, 309,16216 racemos planta $^{-1}$ (Tabela 2).

$\mathrm{Na}$ análise destrutiva a cultivar $\mathrm{MB}$ apresentou valor estatisticamente maior que os outros acessos para massa fresca total (Tabela 5). O menor valor foi obtido no acesso GUR que apresentou diferença estatística apenas das 
cultivares MB e MR. A cultivar MB também apresentou os maiores valores de massa seca total das folhas (MSTF) e massa seca total dos ramos (MSTR), o que resultou em maiores valores de massa seca total (MST) (Tabela 6).

Tabela 5. Valores médios das variáveis massa fresca total (MFT), massa fresca das folhas (MFF), massa fresca das inflorescências (MFI), massa fresca dos ramos (MFR), número de folhas (NF) e número de inflorescências (NI), de quatro acessos e de duas cultivares de manjericão (AC). Gurupi-TO, 2012.

\begin{tabular}{|c|c|c|c|c|c|c|}
\hline \multirow[t]{2}{*}{$\mathrm{AC}$} & MFT & MFF & MFI & MFR & \multirow[t]{2}{*}{ NF } & \multirow[t]{2}{*}{ NI } \\
\hline & \multicolumn{4}{|c|}{ 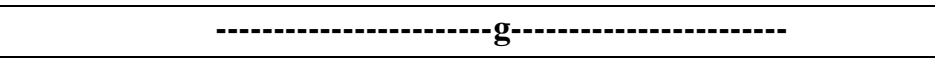 } & & \\
\hline MAL & $343,95 \mathrm{bc}$ & $132,39 \mathrm{c}$ & $74,47 \mathrm{ab}$ & $137,09 \mathrm{bc}$ & $5868,02 \mathrm{a}$ & $344,69 \mathrm{a}$ \\
\hline GUR & $245,15 \mathrm{c}$ & $100,54 \mathrm{c}$ & $48,78 \mathrm{~b}$ & $95,84 \mathrm{c}$ & $4075,50 \mathrm{a}$ & $241,94 \mathrm{ab}$ \\
\hline FPS & $325,28 \mathrm{bc}$ & $138,56 \mathrm{bc}$ & $65,67 \mathrm{~b}$ & $121,05 \mathrm{bc}$ & $4807,38 \mathrm{a}$ & $290,29 \mathrm{a}$ \\
\hline MVE & $281,50 \mathrm{c}$ & $109,37 \mathrm{c}$ & $74,11 \mathrm{ab}$ & $98,02 \mathrm{bc}$ & 4806,10 a & $313,02 \mathrm{a}$ \\
\hline $\mathrm{MB}$ & 891,61 a & 443,16 a & $113,87 \mathrm{a}$ & $334,58 \mathrm{a}$ & 3445,31 a & $213,21 \mathrm{ab}$ \\
\hline MR & $534,99 \mathrm{~b}$ & $240,86 \mathrm{~b}$ & $89,12 \mathrm{ab}$ & $205,00 \mathrm{~b}$ & $3487,31 \mathrm{a}$ & $84,63 \mathrm{~b}$ \\
\hline
\end{tabular}

* Médias seguidas pela mesma letra na coluna não diferem entre si pelo teste de Tukey $(\mathrm{P}>0,05)$

MAL - Monte Alegre-GO; GUR - Gurupi-TO; FPS - Faz. Ponta da Serra; MVE - Montes Verdes; MB - Cv. Maria Bonita; e MR - Manjericão Roxo Comercial.

Tabela 6. Valores médios das variáveis número de ramos (NR), massa seca total das folhas (MSTF), massa seca total das inflorescências (MSTI), massa seca total dos ramos (MSTR), massa seca total (MST), de quatro acessos e de duas cultivares de manjericão (AC).Gurupi-TO, 2012.

\begin{tabular}{|c|c|c|c|c|c|}
\hline \multirow[t]{2}{*}{$\mathbf{A C}$} & \multirow[t]{2}{*}{ NR } & MSTF & MSTI & MSTR & MST \\
\hline & & \multicolumn{4}{|c|}{ 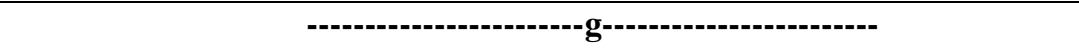 } \\
\hline MAL & $656,58 \mathrm{a}$ & $27,32 \mathrm{~b}$ & $36,48 \mathrm{~b}$ & $41,70 \mathrm{~b}$ & $100,97 \mathrm{bc}$ \\
\hline GUR & $450,56 \mathrm{a}$ & $21,13 b$ & $25,27 \mathrm{~b}$ & $28,62 \mathrm{~b}$ & $75,01 \mathrm{c}$ \\
\hline FPS & $637,25 \mathrm{a}$ & $25,46 \mathrm{~b}$ & $38,95 \mathrm{ab}$ & $54,65 \mathrm{ab}$ & $119,06 \mathrm{abc}$ \\
\hline MVE & $473,04 \mathrm{a}$ & $31,10 \mathrm{~b}$ & $58,38 \mathrm{a}$ & $34,74 \mathrm{~b}$ & $124,21 \mathrm{abc}$ \\
\hline MB & $381,94 \mathrm{a}$ & $73,32 \mathrm{a}$ & $30,41 \mathrm{~b}$ & $79,23 \mathrm{a}$ & $182,97 \mathrm{a}$ \\
\hline MR & $480,06 \mathrm{a}$ & $36,86 \mathrm{~b}$ & $20,75 \mathrm{~b}$ & $31,71 \mathrm{~b}$ & $141,51 \mathrm{ab}$ \\
\hline
\end{tabular}

* Médias seguidas pela mesma letra na coluna não diferem entre si pelo teste de Tukey $(\mathrm{P}>0,05)$.

MAL - Monte Alegre-GO; GUR - Gurupi-TO; FPS - Faz. Ponta da Serra; MVE - Montes Verdes; MB - Cv. Maria Bonita; e MR - Manjericão Roxo Comercial.

O maior valor de MFT apresentada na cultivar MB em relação aos demais acessos é consequência do acúmulo de água e maior eficiência fotossintética de seus tecidos (PAIVA \& OLIVEIRA, 2006). Na cultivar MB observou-se uma produção de $182,97 \mathrm{~g} \mathrm{planta}^{-1}$ de massa seca total, estimando uma produtividade de aproximadamente $10978 \mathrm{~kg} \mathrm{ha}^{-1}$. Em trabalhos desenvolvidos por Blank et al. (2007) com genótipos de manjericão foi constatado que a cultivar Maria Bonita destacou-se em relação aos outros genótipos avaliados com maior produtividade de massa seca de folhas e inflorescências.

Deve ser ressaltada a importância do programa de melhoramento e adaptação ao cultivo nas cultivares MB e $\mathrm{MR}$, enquanto que nos acessos silvestres não foi realizado um trabalho de adaptação às condições de cultivo.

As cultivares MB e MR destacaram com maiores valores de massa fresca da folha (MFF), consequência de sua área foliar maior (Tabela 5). Como foi anteriormente abordado neste trabalho, foi observado nas cultivares MB e MR folhas mais largas, enquanto nos acessos silvestres foi observado folhas mais estreitas.

Segundo Blank et al. (2007), as folhas do cultivar Maria Bonita possui comprimento médio de $6,5 \mathrm{~cm}$ e largura de 2,8 $\mathrm{cm}$. Este fato confere elevada eficiência fotossintética com armazenamento de fotoassimilados influenciando no aumento da massa fresca total das plantas (BENINCASA, 2004).
Fernandes et al. (2004) avaliaram a produtividade em função da massa fresca de duas espécies de manjericão e constataram que a espécie de folha estreita apresentou menor massa fresca acumulada do que as plantas da espécie de folha larga.

A partir dos dados de crescimento podem se ampliar os conhecimentos a respeito da biologia da planta, permitindo o desenvolvimento de técnicas de manejo das espécies ou estimando as causas de variação de crescimento entre plantas geneticamente diversas (CASTRO et al., 1999).

Quanto à massa fresca da inflorescência (MFI), a cultivar MB obteve maior valor, não apresentando diferença da cultivar MR e dos acessos MVE e MAL. O menor valor de massa fresca da inflorescência foi obtido no acesso GUR, o que está relacionado com o menor tamanho de seus racemos. Desta forma, apesar do acesso GUR ter apresentado uma quantidade de racemos superior a cultivar MB, a sua massa fresca de inflorescências foi inferior (Tabela 5).

$\mathrm{O}$ acesso MAL obteve o maior valor de número de inflorescências (NI) com 344,69 racemos planta ${ }^{-1}$, apresentando diferença $(\mathrm{P}<0,05)$ apenas da cultivar MR. Apesar de apresentar um valor superior quanto ao NI, o acesso MAL não se destacou quanto à massa fresca da inflorescência, evidenciando que o tamanho dos racemos interferiu nesta resposta, devido ter apresentado o menor tamanho de racemos. Os racemos da cultivar MR eram em 
menor número, porém consistiam em estruturas de tamanho maior (Tabela 5).

A massa fresca dos ramos (MFR) foi maior na cultivar MB com 334,58 g planta $^{-1}$, apesar de ter apresentado a menor quantidade de ramos (381,94 ramos planta $^{-1}$ ) (Tabela 5). Segundo observações de campo, foi constatado que os ramos da cultivar MB apresentaram diâmetro maior do que os outros acessos.

No número de folhas (NF), não houve diferença entre os acessos. Entretanto, o acesso MAL obteve o maior valor de NF $\left(5868,02\right.$ folhas planta $\left.^{-1}\right)$, embora o tamanho das folhas no acesso MAL tenha sido menor, o que resultou em valores menores de MFF. O inverso foi observado para a cultivar $\mathrm{MB}$, que apresentou o menor valor de NF (3445,31 folhas planta $\left.^{-1}\right)$, no entanto, apresentou folhas maiores e consequentemente, maiores valores de MFF (Tabela 5).

Diante do exposto, deve ser ressaltada a importância de estudos sobre aspectos agronômicos do manjericão no Estado do Tocantins que são escassos. A cultura do manjericão pode ser uma alternativa para os agricultores da região e fonte de renda para os proprietários de pequenas áreas.

\section{CONCLUSÃO}

As cultivares MB e MR apresentaram melhor desempenho na análise de crescimento e na análise destrutiva e podem ser indicadas para o cultivo do manjericão no Estado do Tocantins. Deve ser destacado os maiores valores obtidos em MFT e MST na cultivar MB, o que permite obter uma maior produtividade e rentabilidade da cultura. Entretanto, outros ensaios devem ser realizados para verificar a consistência dos resultados e a adaptação às condições edafoclimáticas em diferentes anos agrícolas.

A caracterização e a preservação dos acessos silvestres coletados tem importância para a sua utilização no programa de melhoramento genético do manjericão para adaptação a estresses bióticos e abióticos.

\section{AGRADECIMENTOS}

Ao Conselho Nacional de Desenvolvimento Científico e Tecnológico (CNPq), à Coordenação de Aperfeiçoamento de Pessoal de Nível Superior (Capes), pela concessão de bolsas e apoio financeiro.

\section{REFERÊNCIAS BIBLIOGRÁFICAS}

Benincasa, M. M. P. Análise de crescimento de plantas: noções básicas. Jaboticabal: FUNEP, 2004. 41p.

Blank, A. F. Carvalho Filho, L. S.; Santos Neto, A. L. dos; Alves, P. B.; Arrigoni-Blank, M. de F.; Silva-Mann, R.; Mendonça, M. da C. Caracterização morfológica e agronômica de acessos de manjericão e alfavaca. Horticultura Brasileira, v. 22, n. 1, p. 113-116, 2004.

Blank, A. F.; Fontes, S. M.; Oliveira, A. dos S.; Mendonça, M. da C.; Silva-Mann, R.; Arrigoni-Blank, M. de F. Produção de mudas, altura e intervalo de corte em melissa. Horticultura Brasileira, v. 23, n. 3, p. 780784, 2005
Blank, A. F.; Souza, E. M. de; Arrigoni-Blank, M. de F. Novas Cultivares - Maria Bonita: cultivar de manjericão tipo linalol. Pesquisa Agropecuária Brasileira, v. 42, n. 12, p. 1811-1813, 2007.

Castro, H. G.; Casali, V. W. D.; Cecon, P. R. Crescimento inicial e épocas de colheita em seis acessos de Baccharis myriocephala D.C. Revista Brasileira de Plantas Medicinais, v. 2, n. 1, p. 1-6, 1999.

Castro, H. G. de; Ferreira, F. A.; Silva, D. J. H. da; Ribeiro Júnior, J. I. Análise do crescimento de acessos de mentrasto (Ageratum conyzoides L.) em dois ambientes. Revista Ciência Agronômica, v. 37, n. 1, p. 44-49, 2006.

Chagas, J. H.; Pinto, J. E. B. P.; Bertolucci, S. K. V.; Santos, F. M. do. Produção de biomassa e teor de óleo essencial em função da idade e época de colheita em plantas de hortelã-japonesa. Acta Scientiarum. Agronomy, v. 33, n. 2, p. 327-334, 2011 .

Fernandes, P. C.; Facanali, R.; Teixeira, J. P. F.; Furlani, P. R.; Marques, M. O. M. Cultivo de manjericão em hidroponia e em diferentes substratos sob ambiente protegido. Horticultura Brasileira, v. 22, n. 2, p. 260264, 2004.

Lourenzani, A. E. B. S.; Lourenzani, W. L.; Batalha, M. O. Barreiras e oportunidades na comercialização de plantas medicinais provenientes da agricultura familiar. Informações Econômicas, v. 34, n. 3, p. 15-25, 2004.

Luz, J. M. Q.; Morais, T. P. S.; Blank, A. F.; Sodré, A. C. B.; Oliveira, G. S. Teor, rendimento e composição química do óleo essencial de manjericão sob doses de cama de frango. Horticultura Brasileira, v. 27, n. 3, p. 349-353, 2009.

May, A.; Bovi, O. A.; Maia, N. B.; Barata, L. E. S.; Souza, R. de C. Z. de; Souza, E. M. R. de; Moraes, A. R. A. de; Pinheiro, M. Q. Basil plants growth and essential oil yield in a production system with successive cuts. Bragantia, v. 67, n. 2, p. 385-389, 2008.

Paiva, R.; Oliveira, L. M. Análise de crescimento. In: Fisiologia e produção vegetal. Lavras: Editora UFLA, 2006. p. 95-100.

Pinto, D. S.; Tomaz, A. C. de A.; Tavares, J. F.; TenórioSouza, F. H.; Dias, C. da S.; Braz-Filho, R.; Da-Cunha, E. V. L. Secondary metabolites isolated from Richardia brasiliensis Gomes (Rubiaceae). Revista Brasileira de Farmacognosia, v. 18, n. 3, p. 367-372, 2008.

Ribeiro júnior, J. I.; Melo, A .L. P. Guia prático para utilização do SAEG. Viçosa: Editora UFV, 2009. 287p.

Rosado, L. D. S.; Pinto, J. E. B. P.; Botrel, P. P.; Bertolucci, S. K. V.; Niculau, E. dos S.; Alves, P. B. Influência do processamento da folha e tipo de secagem no teor e composição química do óleo essencial de manjericão cv. Maria Bonita. Ciência e Agrotecnologia, v. 35, n. 2, p. 291-296, 2011.

Silva, F. da; Santos, R. H. S.; Andrade, N. J. de; Barbosa, L. C. A.; Casali, V. W. D.; Lima, R. R. de; Passarinho, R. V. de M. Basil conservation affected by cropping season, harvest time and storage period. Pesquisa Agropecuária Brasileira, v. 40, n. 4, p. 323-328, 2005. 